\title{
Genetic Polymorphisms on 4q21.1 Contributed to the Risk of Hashimoto's Thyroiditis
}

\author{
Dachao Mo, Junjiu Li, Liang Peng, Zhiyuan Liu, Jieyun Wang, and Jiru Yuan
}

Background: Hashimoto's thyroiditis (HT) is a common autoimmune disease characterized by lymphoid infiltration of the thyroid gland, including both T- and B-cells. Early studies have shown that HT is a complex disorder affected by both environmental and genetic factors. Recently, the single nucleotide polymorphism (SNP) rs2276886 associated with the CXCL9 gene was identified as associated with autoimmune thyroid disease susceptibility in Japanese populations. The aim of the present study was to validate this result for HT in a Chinese Han population. Methods: Study subjects, including 688 HT cases and 1456 healthy controls, were recruited, and 10 SNPs located within the CXCL9 gene were genotyped. Genetic association analyses were performed by fitting logistic models. Bioinformatics tools, including RegulomeDB and GTEx were utilized to investigate the functional consequences of the SNPs found to be significantly associated with HT.

Results: SNP rs2276886 was identified as significantly associated with the risk of HT (odds ratio $[\mathrm{OR}]=1.25$, $p=0.0006$ ). No significant expression quantitative trait loci (eQTL) signals could be identified for $C X C L 9$. Significant eQTL signals were found for other genes, including ART3, CXCL10, CXCL11, NAAA, PPEF2, and SCARB2. This SNP physically maps to the CXCL9 gene region; however, further bioinformatic analyses indicated that this SNP might be associated with the gene $N A A A$.

Conclusions: The rs2276886 SNP was found to be significantly associated with HT susceptibility. However, our findings suggest that this SNP which maps to the chromosomal region 4q21.1 likely effects the NAAA gene (as opposed to the CXCL9 gene), but still contributes to the susceptibility to HT in Han Chinese populations.

Keywords: Hashimoto's thyroiditis, single nucleotide polymorphisms, 4q21.1, NAAA, CXCL9

\section{Introduction}

A $S$ ONE OF THE autoimmune thyroid diseases, Hashimoto's thyroiditis (HT) is a common autoimmune disease that is characterized by lymphoid infiltration of the thyroid gland, including both $\mathrm{T}$ and $\mathrm{B}$ cells (Ajjan and Weetman, 2015). The etiology of HT involves excessively stimulated CD4 ${ }^{+}$T cells (Pyzik et al., 2015), and a decreased sensitivity of $\mathrm{CD}^{+} \mathrm{T}$ cells to the inhibitory effects of TGF $\beta$ may be another potential mechanism (Mirandola et al., 2011). Chronic inflammation in the thyroid gland during HT may impair thyroid function and cause hypothyroidism (depression), myxedema (heart failure), and goiter (painless swelling of the thyroid gland in the front of the neck). With this severe illness, few prevention measures can be prescribed for HT because of a lack of knowledge about its pathogenesis. Along with an elevation in the incidence of HT, studies on HT's pathogenesis have gradually increased.

Numerous studies have shown that the etiology of HT involves many factors such as genetic susceptibility (Aka- hane et al., 2016), diet (Xu et al., 2016) and infection (Caselli et al., 2012), but the true pathogenesis of HT has not yet been discovered. A higher sibling risk ratio in Korean (Villanueva et al., 2003) and German (Dittmar et al., 2011) populations suggests that there is a genetic predisposition to HT (Hwangbo and Park, 2018). With the rapid development of high-throughput sequencing, however, more and more susceptibility variants of complex diseases have been identified (Guan et al., 2014; Liu et al., 2014; Guan et al., 2016). However, current study results for HT account for only a small percentage of the estimated heritability in a few populations, and a corresponding biological interpretation is lacking and, therefore, the molecular mechanism of HT remains poorly understood.

Most recently, a single nucleotide polymorphism (SNP) (rs2276886) within the CXCL9 (MIG) gene was newly identified to be associated with HT susceptibility in Japanese populations (Akahane et al., 2016). Chemokines such as CXCL9 are a class of cytokine proteins that act as signaling molecules, regulating immune and inflammatory responses

Department of General Surgery, Dongguan Tungwah Hospital, Dongguan, China. 
and modulating cell migration properties and localization of target cells such as leucocytes (Moser et al., 2004). The chemokine CXCL9, also known as MIG (a T cell chemoattractant monokine induced by IFN-g), which is commonly produced by local cells in inflammatory lesions, plays important roles in several autoimmune diseases (Smit et al., 2003; Tokunaga et al., 2018).

For example, CXCL9, which is highly expressed in the valvular tissue of chronic rheumatic heart disease patients, mediates the development of the disease by recruitment of $T$ cells (Fae et al., 2013). Furthermore, increases in circulating CXCL9 have been found in patients with thyroiditis and hypothyroidism (Antonelli et al., 2011). Recently, Luo et al. (2018) found that increased levels of MRP14, which is induced by the IL-1beta/MAPK pathway, mediating TFCderived chemokine secretion, including CXCL9 in HT patients, play a significant role in the mechanism of HT. Although many studies have hinted at the potential association between CXCL9 and HT, the underlying molecular mechanisms are largely unknown.

The polymorphisms of genes, including chemokines, have been conclusively identified as risk factors in the pathogenesis of HT (Akahane et al., 2016). A relationship between polymorphisms of CXCL9 and HT in the Japanese population has been shown (Akahane et al., 2016). However, the contributions of CXCL9 to the etiology and pathogenesis of HT have not yet been fully elucidated.

To date, the roles of $C X C L 9$ in HT susceptibility in the Han Chinese population have not been investigated. Considering the genetic heterogeneity in different ethnic populations, the exploration of a potential association between the CXCL9 gene and HT among other independent populations is needed to confirm the previous results, which may shed light on its underlying mechanisms. Therefore, to determine whether the CXCL9 gene is associated with susceptibility to HT in Han Chinese populations, we conducted a hospital-based case/control study, which is the first study to evaluate the relationship between the CXCL9 gene and HT in a Han Chinese population.

\section{Methods}

\section{Study subjects}

Study subjects, including 688 HT cases and 1456 healthy controls, were recruited from Dongguan Tungwah Hospital between June 2011 and January 2019. Unrelated female individuals with a reported ancestry of Han Chinese were included in this study. Diagnosis of HT was based on (1) enlargement of the thyroid, (2) signs of hypoechogenicity and a nonhomogeneous texture on ultrasound images, and (3) a high level of either antithyroid peroxidase (TPO) or antithyroglobulin $(\mathrm{Tg})$, with or without clinical and biochemical hypothyroidism. Individuals with a history of thyroid cancer and/or previous thyroid surgery were excluded from this study. Individuals with normal thyroid function and those who had negative results for thyroid autoantibodies were enrolled as controls.

Any individuals with the existence of any comorbid cardiac, autoimmune, infectious, musculoskeletal, or malignant disease, or a recent history of operation or trauma were excluded from this study. Thyroid function tests were performed using The Roche Diagnostics COBAS 6000 E601
Module Immunochemistry Analyzer (Roche, Basal, Switzerland), and five indicators, including namely, free triiodothyronine, free thyroxine, thyrotropin, anti-TPO, and anti-Tg, were measured in this study. Demographic information such as age, family history of HT, smoking, and alcohol drinking status were collected based on structured questionnaires. Peripheral blood was obtained from each study subject. This study protocol was approved by the Medical Ethics Committee of Dongguan Tungwah Hospital. Written informed consent was obtained from all of the participants.

\section{SNP selection and genotyping}

Based on 1000 genomes of Chinese Han Beijing data, SNPs with a minor allele frequency $(\mathrm{MAF}) \geq 0.05$ located within the gene region of CXCL9 were included in this study. A total of 10 SNPs were selected for genotyping. We extracted the genomic DNA from peripheral blood leukocytes using the Genomic DNA Kit (Axygen Scientific, Inc., CA). The Sequenom MassARRAY RS1000 system (Sequenom, San Diego, CA) was used for SNP genotyping. Typer Analyzer software was utilized for genotype data processing (Guan et al., 2012). Cases and controls were blind labeled during the genotyping process. Five percent of our samples were randomly chosen for replicating and the genotypes were reported to be $100 \%$ the same as in the previous sequencing run.

\section{Statistical analyses}

Logistic models were fitted for each SNP to examine their potential genetic association signals. Age was included as a covariate. In addition, we have also analyzed the potential association between the significant SNP and the thyroid function indicators. Linear models were fitted. Plink (Chang et al., 2015) was utilized for both logistic and linear regression analyses. Linkage disequilibrium (LD) structure was estimated using Haploview (Barrett et al., 2005). Haplotypebased association analyses were performed using Plink (Chang et al., 2015). Multiple comparisons were corrected by Bonferroni and the threshold of $P$ values used for genetic association analyses was $0.05 / 10=0.005$.

\section{Bioinformatics analyses}

Bioinformatics tools were utilized to investigate the functional consequences of the significant SNPs identified from the genetic association analyses. RegulomeDB (Xie et al., 2013) was used to examine the effects of the SNP on gene expression regulation. RegulomeDB is a publicly available database that annotates the genetic variants by integrating data obtained from the ENCODE project. It graded each SNP for its potential role played in the regulation of gene expression. In addition, expression quantitative trait loci (eQTL) data in multiple human tissues were extracted from the GTEx database (GTEx Consortium, 2013) for targeted SNPs to analyze their potential effects on gene expression.

\section{Results}

\section{Demographic information}

The demographic information about our study subjects is summarized in Table 1. No significant differences were 
Table 1. Characteristics of and Clinical Information About Our Study Subjects

\begin{tabular}{lcccc}
\hline & HT cases $(\mathrm{n}=688)$ & Controls $(\mathrm{n}=1456)$ & Statistics & $\mathrm{p}$ \\
\hline Age, years & $41.18 \pm 6.66$ & $41.40 \pm 6.40$ & $T=-0.71$ & 0.480 \\
Family history (\%) & $92(13)$ & $52(4)$ & & \\
$\quad$ Yes & $596(87)$ & $1404(96)$ & $\chi^{2}=70.07$ & $<0.001$ \\
$\quad$ No & & & \\
Smoking (\%) & $36(5)$ & $1392(4)$ & & \\
$\quad$ Yes & $652(95)$ & $130(9)$ & $\chi^{2}=0.56$ & 0.454 \\
$\quad$ No & $65(9)$ & $1326(91)$ & & \\
Alcohol drinking (\%) & $623(91)$ & $3.39 \pm 0.36$ & $\chi^{2}=0.10$ & 0.757 \\
$\quad$ Yes & $4.21 \pm 0.73$ & $13.47 \pm 1.33$ & $T=27.65$ & $<0.001$ \\
$\quad$ No & $15.41 \pm 2.49$ & $1.62 \pm 0.93$ & $T=19.31$ & $<0.001$ \\
$\mathrm{FT}_{3}, \mathrm{pM}$ & $4.85 \pm 0.64$ & $10.93 \pm 7.83$ & $T=93.70$ & $<0.001$ \\
$\mathrm{FT}_{4}, \mathrm{pM}$ & $503.23 \pm 264.28$ & $28.72 \pm 21.86$ & $T=48.85$ & $<0.001$ \\
TSH, mIU/L & $459.41 \pm 260.19$ & & $T=43.35$ & $<0.001$ \\
Anti-TPO, IU/mL & & & \\
Anti-Tg, IU/mL & & & & \\
\hline
\end{tabular}

$\mathrm{FT}_{3}$, free triiodothyronine; FT4, free thyroxine; HT, Hashimoto's thyroiditis; Tg, thyroglobulin; TPO, thyroid peroxidase; TSH, thyrotropin.

obtained for age, smoking, or alcohol drinking status between the HT cases and healthy controls. Significant differences between cases and controls were identified for a family history of HT and all of the five thyroid functional indicators.

\section{Genetic association}

All of the selected SNPs had an MAF greater than 0.01 and were in Hardy-Weinberg equilibrium in our study subjects (Supplementary Table S1). We conducted Hardy-Weinberg equilibrium tests for each SNP in control samples. Among all of these 10 SNPs genotyped in our study, only SNP rs2276886 was identified to be significantly associated with the risk of HT (Table 2). As indicated, the $A$ allele of SNP rs2276886 was found to be significantly associated with the increased risk of HT in our samples (odds ratio $[\mathrm{OR}]=1.25, p=0.0006$ ), and a similar pattern with a significant signal was identified in the genotypic analyses $(p=0.0017)$. The full results of the association analyses are summarized in Supplementary Table S2.

In addition, we have also performed association analyses for SNP rs2276886 and thyroid function indicators stratified by their HT status. No significant association could be identified between SNP rs2276886 and all five indicators in the HT cases and control groups (Supplementary Tables S3). The LD structure based on the genotype data is shown in Figure 1. One 2-SNP LD block (rs72607852-rs75180995) was constructed and results of haplotypic analyses were summarized in Supplementary Table S4. No significant haplotype was identified.

\section{Functional consequences and eQTL signals for SNP rs2276886}

SNP rs2276886 is an intronic variant. We examined its potential functional consequences in RegulomeDB. RegulomeDB has a score system ranging from 1 to 7 , and a lower score usually indicates more functional significance. SNP rs2276886 had a score of $1 \mathrm{~b}$, which indicated that it had significant functional significance. SNP rs2276886 was located at a DNaseI hypersensitivity region and was also found to be located at a transcription factor-binding region. We also examined the eQTL signals for SNP rs2276886 based on data extracted from the GTEx database. Although no significant eQTL signals could be identified for the gene CXCL9 (Supplementary Table S5), a couple of significant eQTL signals could be found for some other genes, including ART3, CXCL10, CXCL11, NAAA, PPEF2, and SCARB2 (Fig. 2). In addition, a significant eQTL signal for SNP rs2276886 in human thyroid tissue could only be found on gene NAAA (Fig. 3).

\section{Discussion}

In the present study we have identified an intronic SNP rs2276886 to be significantly associated with HT. Our

Table 2. Results of Genetic Association Analyses for Single Nucleotide Polymorphism Rs2276886

\begin{tabular}{|c|c|c|c|c|c|c|c|c|c|c|}
\hline & \multicolumn{3}{|c|}{ Genotype } & \multirow[b]{2}{*}{ Statistics } & \multirow[b]{2}{*}{$\mathrm{p}$} & \multicolumn{2}{|c|}{ Allelic } & \multirow[b]{2}{*}{$O R(95 \% C I)$} & \multirow[b]{2}{*}{ Statistics } & \multirow[b]{2}{*}{$\mathrm{p}^{*}$} \\
\hline & $A A$ & $A G$ & $G G$ & & & $\boldsymbol{A}$ & $G$ & & & \\
\hline $\begin{array}{l}\text { HT cases } \\
\quad(n=688)\end{array}$ & $180(26)$ & $326(47)$ & $182(27)$ & & & $686(50)$ & $690(50)$ & & & \\
\hline $\begin{array}{l}\text { Controls } \\
\qquad(n=1456)\end{array}$ & $288(20)$ & $711(49)$ & $457(31)$ & $\chi^{2}=12.74$ & 0.0017 & $1287(44)$ & $1625(56)$ & $1.25(1.10-1.42)$ & $T=3.42$ & 0.0006 \\
\hline
\end{tabular}

Risk allele and significant $p$-values are in bold italics, and the threshold used for the significance of the $p$-value was $0.05 / 10=0.005$. OR refers to the risk allele odds ratio in both groups.

${ }^{*} p$-Values with adjustments for age.

$\mathrm{CI}$, confidence interval; OR, odds ratio. 
FIG. 1. Linkage disequilibrium structure of the selected SNPs. Values of D' are indicated in each square. The darker the color is, the greater the value. SNP, single nucleotide polymorphism.

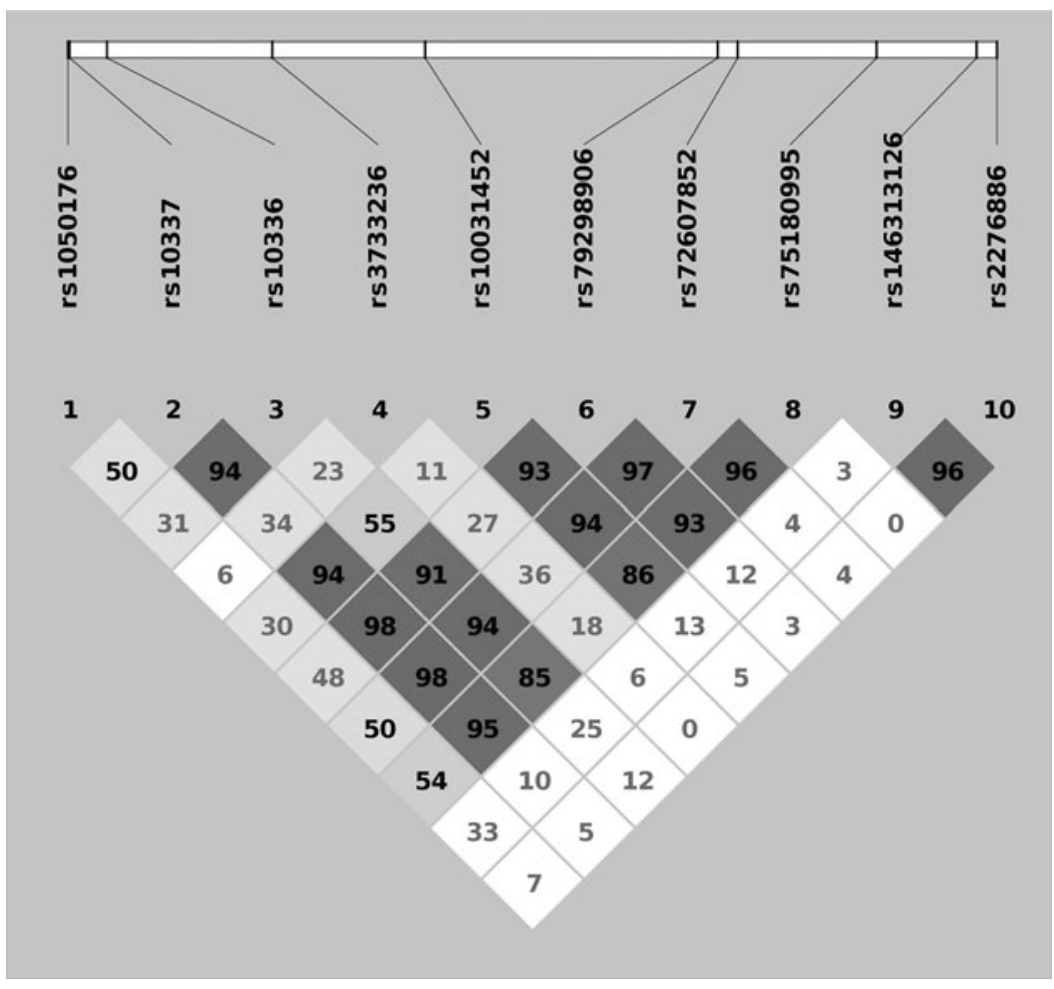

findings replicate those of a study conducted by Akahane et al., based on study subjects with Japanese ancestry. The $A$ allele of rs2276886 was identified to be more frequent in patients in both studies. Considering the genetic similarity between Chinese Han and Japanese, this replication was not surprising. However, one interesting thing to note is our further eQTL analyses based on GTEx. Significant eQTL signals of rs2276886 were not identified for the gene CXCL9 but instead were found for some other genes in the $4 \mathrm{q} 21.1$ region, including ART3, CXCL10, CXCL11, NAAA, PPEF2, and $S C A R B 2$, which are located within a $\pm 200 \mathrm{~kb}$ window of rs2276886.
We originally mapped SNP rs2276886 to the gene CXCL9 based on the physical location of this SNP (the SNP rs2276886 is located within the genetic region of CXCL9). This SNP gene mapping strategy based on physical location is a standard method used by most of the SNP genotyping microarray products and thus has been utilized by most of the previously published genome-wide association studies (GWAS). On the other hand, knowledge of eQTL signals might enable us to perform SNP gene mapping in a more accurate way. Instead of using the traditional mapping strategy based on physical location, we could map SNPs to genes based on their function (functional mapping), and thus

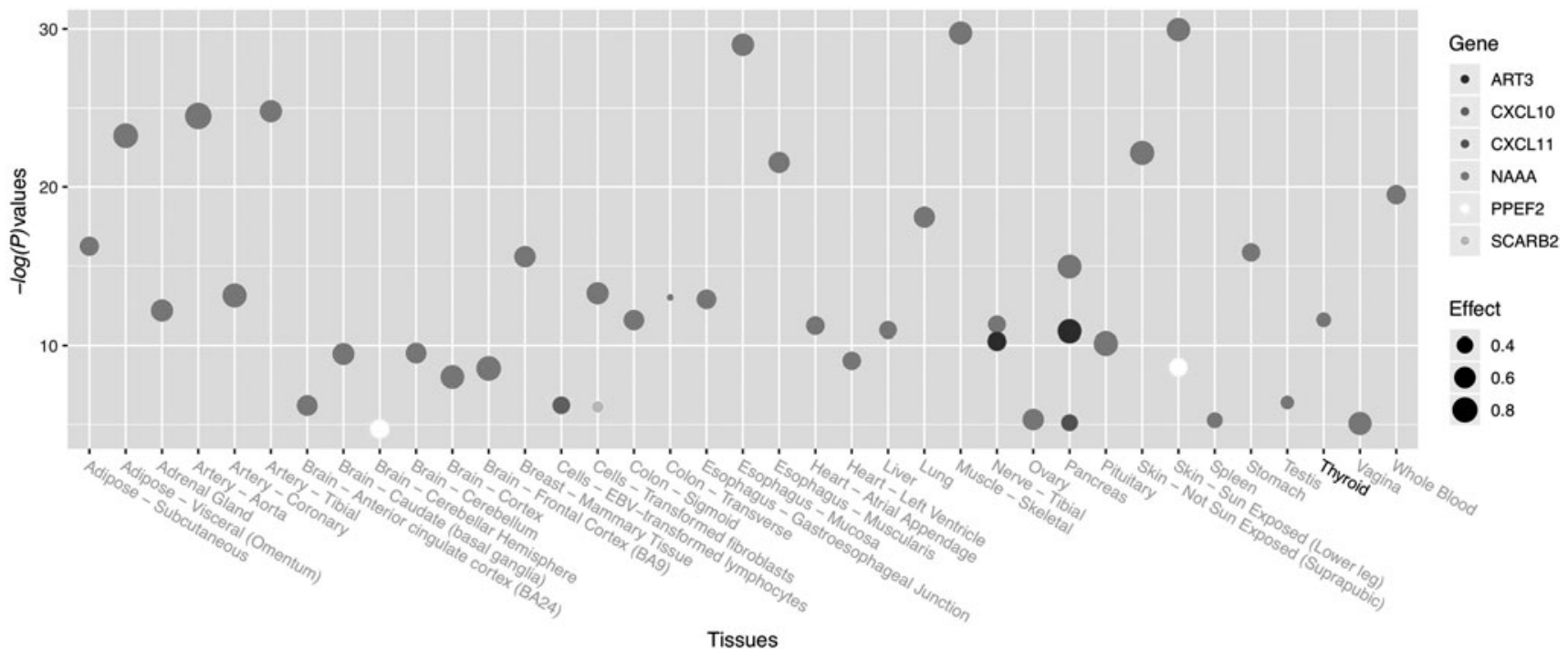

FIG. 2. Significant eQTL signals for SNP rs2276886 on multiple genes. eQTL, expression quantitative trait loci. 


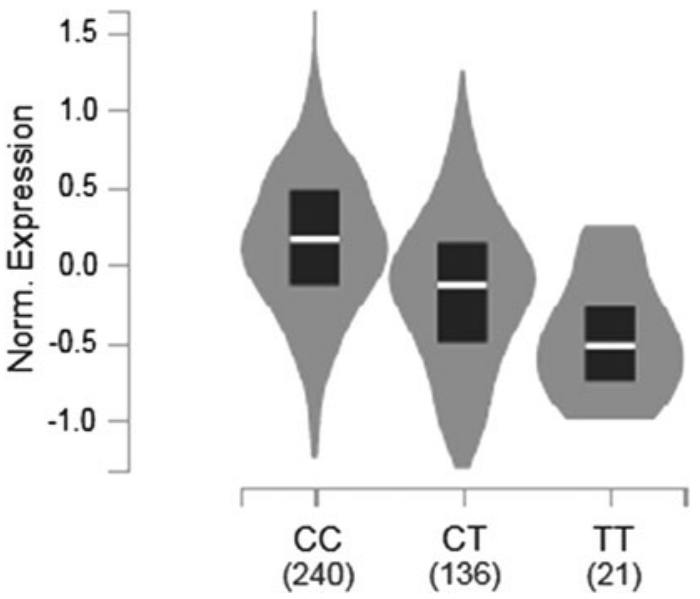

FIG. 3. Expression levels of the gene $N A A A$ in tissues of the thyroid for different genotypes of SNP rs2276886. Violin plots are based on the gene expression levels showed by the gray shade. The mean expression levels are showed by the white line, and $25 \%$ and $75 \%$ quantiles are showed by the black box.

the effect of SNP rs2276886 on the risk of HT was derived from a gene other than $C X C L 9$.

Rs2276886 had a RegulomeDB score of $1 \mathrm{~b}$, which indicated that this SNP was located in a functionally significant genomic region and has a high possibility to be a SNP with functional consequences. Nevertheless, we need to be careful in interpreting these results based on bioinformatics analyses. As is known, a great many SNPs have significant eQTL data in GTEx simply because they represent points along an eQTL association peak.

We cannot simply consider the $p$-value as indicating that the SNP is a driver for the eQTL. Instead, we should further question whether the full range of eQTL data across a genomic range significantly colocalizes with a disease association peak. Functional studies in the future are needed to validate these eQTL signals. Thus, it is difficult to draw solid conclusions only from SNP-based association analysis (Yang et al., 2013; Chen et al., 2015; Guan et al., 2015; Zhang et al., 2015, 2018; Jia et al., 2016; Han et al., 2018), and further functional studies focusing on the associated SNP are desirable to elucidate the role of the SNP in the susceptibility of HT.

Among the six genes that showed eQTL signals for SNP rs2276886, NAAA is particularly interesting. Among 42 significant eQTL signals of rs2276886, 35 were obtained for $N A A A$ from different human tissues, and what is more important is that a significant eQTL signal from human thyroid tissue was only identified for NAAA. In addition, SNP rs2276886 is located $\sim 50 \mathrm{~kb}$ upstream of $N A A A$, which is a hot spot for regulatory regions of genes.

$N A A A$ encodes an $\mathrm{N}$-acylethanolamine-hydrolyzing enzyme that hydrolyzes bioactive $N$-acylethanolamines to fatty acids and ethanolamine. The primary structure of NAAA exhibits $33-35 \%$ amino acid identity to acid ceramidase, which is a lysosomal enzyme hydrolyzing ceramide into fatty acids and sphingosine (Tsuboi et al., 2007). Previous GWAS have mapped NAAA to several human traits, including blood protein levels (Suhre et al., 2017; Sun et al., 2018), cerebrospinal fluid biomarker levels (Sasayama et al., 2017), and coronary artery calcified atherosclerotic plaques in type 2 diabetes (Divers et al., 2017). However, currently, there is no direct connection between NAAA and HT.
In the future, a thorough scan for genetic associations between polymorphic markers in NAAA and HT is necessary, and more biomedical research focusing on the pathological mechanisms of HT are needed.

Our study suffers from several limitations. First, only women were included in this study. Although this femaleonly strategy makes the sample enrollment process more efficient because of the high female:male ratio in HT cases, it in some ways hindered the generalization of the results obtained from this study. Studies with a more balanced gender distribution are needed in the future. Second, in the present study, only common polymorphisms were included. This strategy efficiently lowered the experimental cost but also dropped many low-frequency and rare variants.

In the future, a sequencing-based study is needed to thoroughly investigate the potential genetic architecture in the 4q21.1 region with regard to HT. Potential population stratification (PS) is another limitation of this study. As a common confounding factor in genetic association mapping, it might cause false-positive results. For the current candidate genebased association study, we could not perform some correction methods such as principal component analysis or genomic control as used in the GWAS scenario. Considering that the Chinese population is a population with extensive genetic heterogeneity, the PS might be even more severe than we thought.

In summary, in the present study, SNP rs2276886 was identified to be significantly associated with HT susceptibility. The SNP was physically mapped to the gene region of CXCL9; however, further bioinformatics analyses have indicated that SNP rs2276886 might be connected to the regulation of the gene NAAA. Our findings suggest that $4 \mathrm{q} 21.1$ might be a susceptible locus for HT and rs2276886 may contribute to the susceptibility to HT in Han Chinese populations. Given the complexity of HT pathology and several limitations present in this study, functional studies based on experimental data and biomedical research focusing on the pathological mechanisms of HT are necessary in the future.

\section{Author Disclosure Statement}

No competing financial interests exist.

\section{Funding Information}

No funding was received for this article.

\section{Supplementary Material}

Supplementary Table S1

Supplementary Table S2

Supplementary Table S3

Supplementary Table S4

Supplementary Table S5

\section{References}

Ajjan RA, Weetman AP (2015) The pathogenesis of Hashimoto's thyroiditis: further developments in our understanding. Horm Metab Res 47:702-710.

Akahane M, Watanabe M, Inoue N, et al. (2016) Association of the polymorphisms of chemokine genes (IL8, RANTES, MIG, IP10, MCP1 and IL16) with the pathogenesis of autoimmune thyroid diseases. Autoimmunity 49:312-319. 
Antonelli A, Ferrari SM, Frascerra S, et al. (2011) Increase of circulating CXCL9 and CXCL11 associated with euthyroid or subclinically hypothyroid autoimmune thyroiditis. J Clin Endocrinol Metab 96:1859-1863.

Barrett JC, Fry B, Maller J, Daly MJ (2005) Haploview: analysis and visualization of LD and haplotype maps. Bioinformatics 21:263-265.

Caselli E, Zatelli MC, Rizzo R, et al. (2012) Virologic and immunologic evidence supporting an association between HHV-6 and Hashimoto's thyroiditis. PLoS Pathog 8:e1002951.

Chang CC, Chow CC, Tellier LC, et al. (2015) Secondgeneration PLINK: rising to the challenge of larger and richer datasets. Gigascience 4:7.

Chen G, Guan F, Lin H, et al. (2015) Genetic analysis of common variants in the HDAC2 gene with schizophrenia susceptibility in Han Chinese. J Hum Genet 60:479-484.

Dittmar M, Libich C, Brenzel T, Kahaly GJ (2011) Increased familial clustering of autoimmune thyroid diseases. Horm Metab Res 43:200-204.

Divers J, Palmer ND, Langefeld CD, et al. (2017) Genomewide association study of coronary artery calcified atherosclerotic plaque in African Americans with type 2 diabetes. BMC Genet 18:105.

Fae KC, Palacios SA, Nogueira LG, et al. (2013) CXCL9/Mig mediates $\mathrm{T}$ cells recruitment to valvular tissue lesions of chronic rheumatic heart disease patients. Inflammation 36 : 800-811.

GTEx Consortium (2013) The genotype-tissue expression (GTEx) project. Nat Genet 45:580-585.

Guan F, Li L, Qiao C, et al. (2015) Evaluation of genetic susceptibility of common variants in CACNA1D with schizophrenia in Han Chinese. Sci Rep 5:12935.

Guan F, Zhang B, Yan T, et al. (2014) MIR137 gene and target gene CACNA1C of miR-137 contribute to schizophrenia susceptibility in Han Chinese. Schizophr Res 152:97-104.

Guan F, Zhang C, Wei S, et al. (2012) Association of PDE4B polymorphisms and schizophrenia in Northwestern Han Chinese. Hum Genet 131:1047-1056.

Guan F, Zhang T, Li L, et al. (2016) Two-stage replication of previous genome-wide association studies of AS3MT-CNNM2NT5C2 gene cluster region in a large schizophrenia casecontrol sample from Han Chinese population. Schizophr Res 176:125-130.

Han W, Zhang TX, Ni T, et al. (2018) Relationship of common variants in CHRNA5 with early-onset schizophrenia and executive function. Schizophr Res 206:407-412.

Hwangbo Y, Park YJ (2018) Genome-wide association studies of autoimmune thyroid diseases, thyroid function, and thyroid cancer. Endocrinol Metab (Seoul) 33:175-184.

Jia X, Zhang T, Li L, et al. (2016) Two-stage additional evidence support association of common variants in the HDAC3 with the increasing risk of schizophrenia susceptibility. Am J Med Genet B Neuropsychiatr Genet 171:1105-1111.

Liu XS, Hou Y, Yan TL, et al. (2014) Dopamine D3 receptorregulated NR2B subunits of N-methyl-d-aspartate receptors in the nucleus accumbens involves in morphine-induced locomotor activity. CNS Neurosci Ther 20:823-829

Luo X, Zheng T, Mao C, et al. (2018) Aberrant MRP14 expression in thyroid follicular cells mediates chemokine secretion through the IL-1beta/MAPK pathway in Hashimoto's thyroiditis. Endocr Connect 7:850-858.
Mirandola P, Gobbi G, Masselli E, et al. (2011) Protein kinase Cepsilon regulates proliferation and cell sensitivity to TGF1beta of CD4+ T lymphocytes: implications for Hashimoto thyroiditis. J Immunol 187:4721-4732.

Moser B, Wolf M, Walz A, Loetscher P (2004) Chemokines: multiple levels of leukocyte migration control. Trends Immunol 25:75-84.

Pyzik A, Grywalska E, Matyjaszek-Matuszek B, Rolinski J (2015) Immune disorders in Hashimoto's thyroiditis: what do we know so far? J Immunol Res 2015:979167.

Sasayama D, Hattori K, Ogawa S, et al. (2017) Genome-wide quantitative trait loci mapping of the human cerebrospinal fluid proteome. Hum Mol Genet 26:44-51.

Smit MJ, Verdijk P, van der Raaij-Helmer EM, et al. (2003) CXCR3-mediated chemotaxis of human $\mathrm{T}$ cells is regulated by a Gi- and phospholipase C-dependent pathway and not via activation of MEK/p44/p42 MAPK nor Akt/PI-3 kinase. Blood 102:1959-1965.

Suhre K, Arnold M, Bhagwat AM, et al. (2017) Connecting genetic risk to disease end points through the human blood plasma proteome. Nat Commun 8:14357.

Sun BB, Maranville JC, Peters JE, et al. (2018) Genomic atlas of the human plasma proteome. Nature 558:73-79.

Tokunaga R, Zhang W, Naseem M, et al. (2018) CXCL9, CXCL10, CXCL11/CXCR3 axis for immune activation-a target for novel cancer therapy. Cancer Treat Rev 63:40-47.

Tsuboi K, Takezaki N, Ueda N (2007) The N-acylethanolaminehydrolyzing acid amidase (NAAA). Chem Biodivers 4:19141925.

Villanueva R, Greenberg DA, Davies TF, Tomer Y (2003) Sibling recurrence risk in autoimmune thyroid disease. Thyroid 13:761-764.

Xie D, Boyle AP, Wu L, et al. (2013) Dynamic trans-acting factor colocalization in human cells. Cell 155:713-724.

Xu C, Wu F, Mao C, et al. (2016) Excess iodine promotes apoptosis of thyroid follicular epithelial cells by inducing autophagy suppression and is associated with Hashimoto thyroiditis disease. J Autoimmun 75:50-57.

Yang H, Zhang B, Zhu J, et al. (2013) 4q22.1 contributes to bone mineral density and osteoporosis susceptibility in postmenopausal women of Chinese Han population. PLoS One 8: e80165.

Zhang B, Guan F, Chen G, et al. (2015) Common variants in SLC1A2 and schizophrenia: association and cognitive function in patients with schizophrenia and healthy individuals. Schizophr Res 169:128-134.

Zhang TX, Zhu L, Ni T, et al. (2018) Voltage-gated calcium channel activity and complex related genes and schizophrenia: a systematic investigation based on Han Chinese population. J Psychiatr Res 106:99-105.

Address correspondence to:

Dachao Mo, MD

Department of General Surgery Dongguan Tungwah Hospital No. 1 Dongcheng East Road Dongguan 523110

China

E-mail: dachamoo@163.com 